\title{
The mammalian target of rapamycin (mTOR) kinase mediates haloperidol-induced cataleptic behavior
}

\author{
Uri Nimrod Ramírez-Jarquín ${ }^{1}$, Neelam Shahani ${ }^{1}$, William Pryor ${ }^{1}$, Alessandro Usiello ${ }^{2,3}$ and Srinivasa Subramaniam (i) ${ }^{1}$
}

\begin{abstract}
The mammalian target of rapamycin (mTOR) is a ubiquitously expressed serine/threonine kinase protein complex (mTORC1 or mTORC2) that orchestrates diverse functions ranging from embryonic development to aging. However, its brain tissue-specific roles remain less explored. Here, we have identified that the depletion of the mTOR gene in the mice striatum completely prevented the extrapyramidal motor side effects (catalepsy) induced by the dopamine 2 receptor (D2R) antagonist haloperidol, which is the most widely used typical antipsychotic drug. Conversely, a lack of striatal mTOR in mice did not affect catalepsy triggered by the dopamine 1 receptor (D1R) antagonist SCH23390. Along with the lack of cataleptic effects, the administration of haloperidol in mTOR mutants failed to increase striatal phosphorylation levels of ribosomal protein pS6 (S235/236) as seen in control animals. To confirm the observations of the genetic approach, we used a pharmacological method and determined that the mTORC1 inhibitor rapamycin has a profound influence upon post-synaptic D2R-dependent functions. We consistently found that pretreatment with rapamycin entirely prevented (in a time-dependent manner) the haloperidol-induced catalepsy, and pS6K (T389) and pS6 (S235/236) signaling upregulation, in wild-type mice. Collectively, our data indicate that striatal mTORC1 blockade may offer therapeutic benefits with regard to the prevention of D2R-dependent extrapyramidal motor side effects of haloperidol in psychiatric illness.
\end{abstract}

\section{Introduction}

Mammalian target of rapamycin (mTOR) exists as the mTOR-mLST8-Raptor complex (mTORC1) and mTOR-mLST8-Rictor complex (mTORC2). It serves as a multifunctional kinase in embryonic development, cancer, diabetes, aging, and neurodegenerative diseases ${ }^{1-3}$. Its role and regulation in nervous system physiology and disease, however, is poorly understood ${ }^{4}$. This represents a major knowledge gap because the malfunction of mTORC1 activity (either by being too high or too low) has been linked to a variety of brain dysfunctions such as

Correspondence: Srinivasa Subramaniam (ssubrama@scripps.edu) 'Department of Neuroscience, The Scripps Research Institute, Florida, Jupiter, Florida 33458, USA

${ }^{2}$ Department of Environmental, Biological, and Pharmaceutical Sciences and Technologies, University of Campania Luigi Vanvitelli, 81100 Caserta, Italy

Full list of author information is available at the end of the article epilepsy, mental retardation, tuberous sclerosis, Huntington's disease (HD), Parkinson's disease (PD), and Alzheimer's disease (AD), all of which affect a specific set of neuronal populations in the brain ${ }^{5-9}$. A detailed understanding of how mTOR is regulated and what role it plays in selective brain regions is important for the development of better intervention strategies.

The brain's striatum is composed of more than 95\% inhibitory medium spiny neurons (MSNs) and it plays an important role in motor, cognitive, psychiatric, and reward behaviors ${ }^{10}$. A spectrum of striatal-dependent motor alterations, such as neuromotor abnormalities (hyper and dyskinetic movement, dysfunctional sensorimotor integration, neurological soft signs and gait and posture deficits) and psychomotor deficits (Parkinsonism, psychomotor slowing and catalepsy) are found in brain disorders including autism spectrum disorder (ASD) and 
schizhophrenia (SCZ). MSN dysfunctions play a critical role in the motor abnormalities seen in HD and PD; however, the molecular mechanisms are unclear. Interestingly, global blocking of mTORC1 signaling with rapamycin affords protection against the pathological and behavioral symptoms associated with $\mathrm{HD}$ and $\mathrm{PD}$ in murine models ${ }^{7,8,11-14}$. However, the striatal-specific roles of mTOR signaling in mental disorders and neurodegenerative disease remains obscure.

Two major types of functionally distinct MSN are recognized, based on the dopamine 1 receptor (D1R) or dopamine 2 receptor (D2R) expression found in the striatum. In association with other receptors (e.g., glutamate, serotonin, and adenosine $\mathrm{A} 1$ and $\mathrm{A} 2 \mathrm{~A}$ receptors), dopamine receptors play critical roles in the processing of sensory, motor, cognitive, and motivational functions ${ }^{15,16}$. Functionally, D1R signaling increases Goolf/adenylyl cyclase/cAMP/PKA signaling in the direct pathway of the basal ganglia, whereas D2R signaling inhibits cAMP/PKA signaling in the indirect pathway ${ }^{17-20}$. Both dopamine D1 and D2 receptor stimulation promote motor activity. Pharmacological inhibition either of D1R or the D2R consistently trigger severe motor deficit and extrapyramidal side effects (EPS) ${ }^{21}$.

Recent studies have indicated that coordinated signaling of both D1R and D2R is responsible for the initiation and execution of motor activity ${ }^{22}$. Importantly, mTOR phosphorylation is selectively increased in the striatum during L-DOPA-induced dyskinesia ${ }^{23}$ and motor learning ${ }^{24}$. However, the genetic evidence for the physiological role of mTOR signaling in the striatum (or its role in D1R versus D2R MSNs signaling) is currently unknown. Using genetic and pharmacological approaches, we investigated the role of mTOR on striatal-mediated motor behaviors under basal and challenged conditions.

\section{Results}

\section{Striatal mTOR regulates motor behaviors}

The role of mTOR signaling in the regulation of striatal motor functions under basal conditions remains unclear. To address this question, we carried out conditional depletion of $m T O R$ in the striatum of adult $m T O R^{f l o x} / f l o x$ mice. We used an AAV1.hSyn.HI.WPRE.SV40 variant expressing Cre-GFP (AAV-Cre-GFP) under the control of human synapsin promoter to deplete $m T O R$ preferentially in striatal neuronal cells ${ }^{25}$. We stereotaxically injected purified virus (AAV-Cre-GFP or AAV-GFP) bilaterally into the striatum of 8 -week-old $m T O R^{\text {flox/flox }}$ mice (Fig. 1a, b). Using Ctip2 (a marker for MSNs), we confirmed that in AAV-Cre-GFP-injected $m T O R^{\text {flox } / \text { flox }}$ mice ( $m$ TOR mutant), mTOR is depleted in the striatum 18 weeks after Cre injection, as expected, but not in AAV-GFP-injected $m T O R^{f l o x / f l o x}$ mice (control) (Fig. 1c-e). To determine the potential influence of neuronal $m T O R$ depletion on cell survival, we estimated the number of cells and ventricular size between $m T O R$ mutant and control mice. We found no gross changes in the number of total cells (Fig. 1e) or the ventricular size of the rostral and caudal striatal regions between $m T O R$ mutant and control mice (Fig. 1f, g). These results indicate that AAV-Cre-GFP injection produces $m T O R$ depletion in the MSNs and does not elicit neurodegenerative-like anatomical phenotype in the striatum.

We next assessed the striatal motor functions in $m T O R$ mutant and control mice 2 weeks after AAV-Cre-GFP or AAV-GFP-control injections. As Cre-recombinase injection in the brain may affect behaviors ${ }^{26}$, we have included an additional control group for Cre: WT [C57BL/6] mice injected with AAV-Cre-GFP (Cre-control) or AAV-GFP (GFP-control) in all of our longitudinal behavioral analyses.

First, we measured locomotor activity using the openfield test (OFT). In the OFT, mTOR mutant, control, and Cre/GFP-control mice are placed individually in faintly lit open-field chambers for $30 \mathrm{~min}$ sessions. The $m T O R$ mutant mice displayed a mild increase in forward locomotion at 14 weeks that was not significantly different at 10 or 18 weeks of age, compared to Cre/GFP-injected control animals (Fig. 1h).

Second, we investigated whether depletion of striatal mTOR impacts on balance and motor coordination, which is regulated by the striatum-cerebellar circuitry, using rotarod ${ }^{27}$. The $m T O R$ mutant showed a decreased trend of motor coordination on the rotarod test compared to the control and Cre/GFP-control groups (Fig. 1i). The mTOR mutant mice display a trend of recovering or compensation on the rotarod test at 14 weeks, but that appears to have been lost at 18 weeks of age (Fig. 1i).

Overall, these results indicate that striatal mTOR plays a modulatory role in locomotion and motor coordination under basal conditions.

\section{Striatal mTOR does not influence D1R-mediated motor effects}

Dopamine regulates motor functions such as locomotion and motor coordination by stimulating two main classes of receptors in the striatum (D1R and D2R $)^{28}$. Considering that striatal mTOR depletion produces motor alterations under basal conditions, we questioned to what extent the D1R signaling-mediated function is affected in $m T O R$ mutant mice. To address this question, we intraperitoneally (i.p.) injected pharmacological modulators that either activate D1R-signaling using SKF81297 $(2.5 \mathrm{mg} / \mathrm{kg}$, i.p.) or inhibit D1R-signaling using SCH23390 $\left(0.1 \mathrm{mg} / \mathrm{kg}\right.$, i.p.), as described in previous studies ${ }^{29-32}$. Injection of SKF81297 (2.5 mg/kg, i.p.), a selective agonist of the D1R receptor, produced robust motor stimulation 


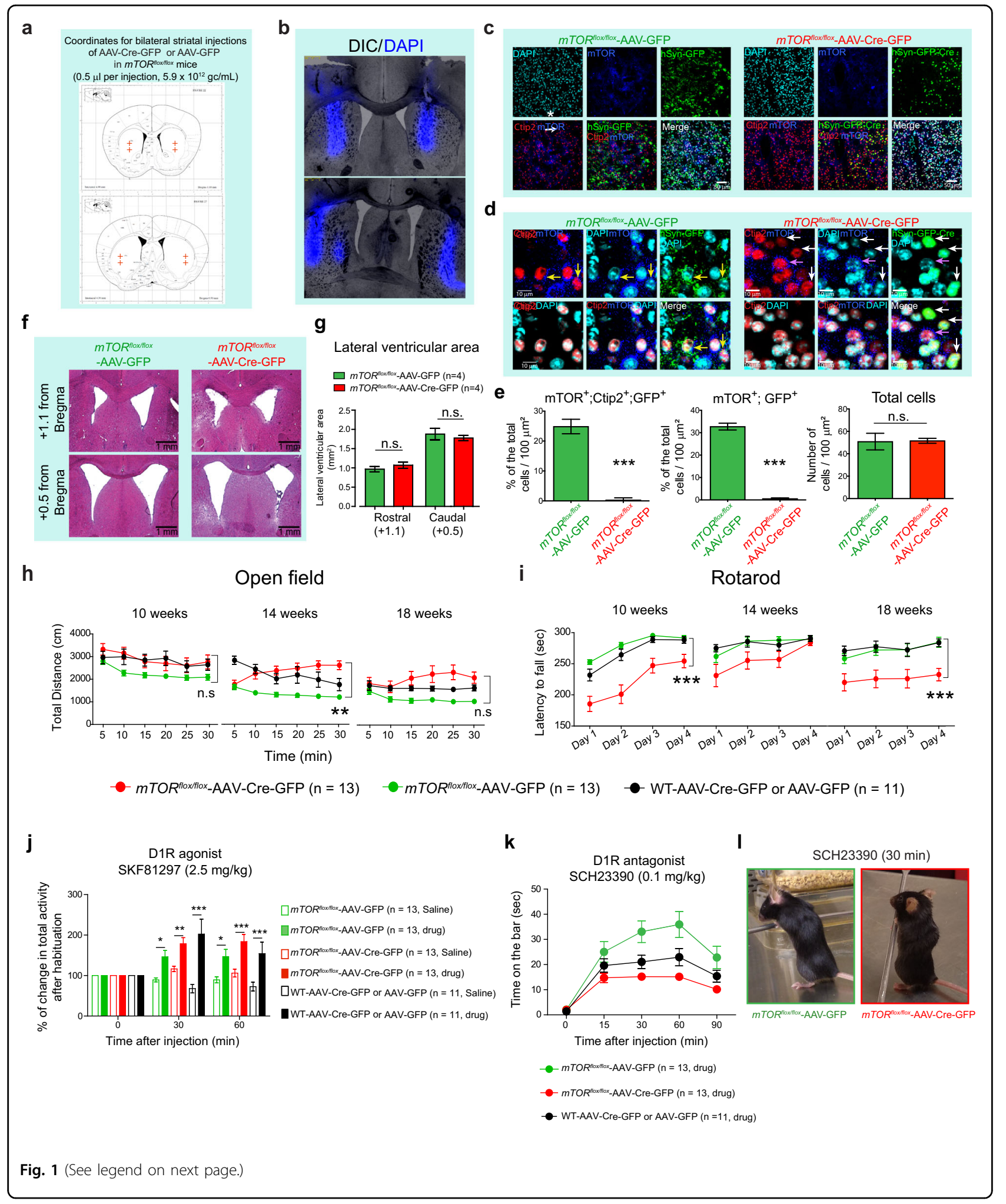

in all animals compared to the saline-administered group. Thus, administration of the D1R agonist induced comparable hyperlocomotion in AAV-Cre-GFP-injected $m T O R^{\text {flox/flox }}$ and control groups $\left(m T O R^{\text {flox/flox }}\right.$ injected with AAV-GFP or WT mice injected with AAV-Cre-GFP or AAV-GFP) when tested at 30 and $60 \mathrm{~min}$ (Fig. 1j). This 
(see figure on previous page)

Fig. 1 Effect of striatal mTOR depletion on motor behaviors. a Schematic representation of the AAV-Cre-GFP or AAV-GFP-injected sites at the indicated coordinates targeting dorsal side of mice striatum. b Representative section showing the DAPI (blue) injection in the striatum using the coordinates in (a). c Confocal images of the striatal brain sections from the mTOR floxflox mice injected with AAV-Cre-GFP or AAV-GFP, showing GFPCre or GFP (green) expression, mTOR (blue), and Ctip2 (red) immunohistochemistry, and nuclear stain, DAPI (cyan). d High magnification of confocal images in (c), showing that in AAV-GFP-injected $m$ mTOR $^{\text {flox flox }}$ mice, Ctip2-positive medium spiny neurons (MSNs) show GFP expression and mTOR immunostaining (yellow arrows). In AAV-Cre-GFP-injected mTOR floxflox mice, Ctip2-positive MSNs express GFP (Cre) but are negative for mTOR immunostaining (white arrows). Some Ctip2-positive MSN negative for GFP (Cre) are positive for mTOR staining (pink arrow). e Quantification for total number of cells identified by DAPI staining, \% of mTOR, Ctip2 and GFP triple-positive neurons and \% of mTOR and GFP double-positive neurons in striatum of the $m T O R^{\text {flox/flox }}$ mice injected with AAV-Cre-GFP or AAV-GFP. Images are representative of five ROls from 4 to 5 sections per animal $(n=4$ mice per group). Percentages were determined by considering the number of DAPI stained nuclei as $100 \%$. All values are mean \pm SEM. n.s. not significant, ${ }^{* * *} P<0.001$, two-tailed Student's $t$ test. $\mathbf{f}$ Representative hematoxylin/eosin-stained sections for rostral ( +1.1 from bregma) and caudal ( +0.5 from bregma) lateral ventricles at the striatal level from the $m T O R^{\text {flox/flox }}$ mice injected with AAV-Cre-GFP or AAV-GFP. $\mathbf{g}$ Quantification of lateral ventricular area from (f). n.s. not significant, two-way ANOVA, Bonferroni post-hoc test (four caudal and four rostral sections were quantified for four mice per group). $\mathbf{h}$, $\mathbf{i}$ Total distance (cm) at the indicated time points in open-field test (OFT) (h) and latency to fall (sec) in rotarod test (i) for the $m_{T O R}$ floxflox injected with AAV-GFP $(n=13$, female $=10$, male $=3$ ), AAV-Cre-GFP $(n=13$, female $=6$, male $=7$ ) or WT mice injected with AAV-GFP or AAV-Cre-GFP $(n=11$, female $=5$, male $=6)$ at 10,14 , and 18 weeks of age. Data are mean \pm SEM. ${ }^{* *} P<0.01$, ${ }^{* * *} P<0.001$, repeated measures twoway ANOVA followed by Bonferroni post-hoc test. j D1R agonist (SKF81297, $2.5 \mathrm{mg} / \mathrm{Kg}$, i.p.)-induced activity in OFT in AAV-Cre-GFP or AAV-GFPinjected $m T O R^{\text {flox/flox }}$ and AAV-Cre-GFP/GFP-injected WT mice. Bar graphs indicates $\%$ of change in total activity after habituation. Data are mean \pm SEM, ${ }^{*} P<0.05,{ }^{* *} P<0.01,{ }^{* * *} P<0.001$, repeated measures two-way ANOVA followed by Bonferroni post-hoc test. $\mathbf{k}$ Quantification of the catalepsy (time on the bar, sec)-induced by D1R antagonist SCH23390 (0.1 mg/Kg, i.p.) in indicated mice groups. Data are mean \pm SEM, $n=11-13$ per group, repeated measures two-way ANOVA followed by Bonferroni post-hoc test. I Representative image of catalepsy in AAV-Cre-GFP or AAV-GFP-injected $m T R^{\text {flox flox }}$ mice treated with SCH23390 (30 min). $m$ TOR $^{\text {floxflox }}$ injected with AAV-GFP $(n=13$, female $=10$, male $=3$ ), AAV-Cre-GFP $(n=13$, female $=6$, male $=7)$ or WT mice injected with AAV-GFP or AAV-Cre-GFP $(n=11$, female $=5$, male $=6)$ were treated with vehicle or drug $(j-I)$.

result indicates that mTOR depletion does not grossly interfere with D1R-mediated motor stimulation.

We next asked whether striatal mTOR plays any role in D1R-mediated catalepsy. Indeed, it has been well established that blocking D1R with its antagonist SCH23390 $(0.1 \mathrm{mg} / \mathrm{kg}$, i.p.) elicits cataleptic behavior (i.e., the animal was unable to correct an externally imposed posturetime spent on the bar $)^{33,34}$. Notably, SCH23390 administration induced a similar cataleptic response in $m T O R$ mutant mice and control groups (Fig. 1k, l). This result indicates that mTOR depletion has no significant effect on D1R antagonist-induced EPS.

Collectively, these data indicate that striatal mTOR does not affect pharmacologically modulated D1R-dependent motor behaviors.

\section{Striatal mTOR promotes D2R inhibition (Haloperidol)- induced cataleptic behavior}

We then investigated whether mTOR depletion modulates pre-and post-synaptic D2R-signalingmediated motor behavior. We administered quinpirole $(0.5 \mathrm{mg} / \mathrm{kg}$, i.p.), a D2R agonist, that by activating the presynaptic $\mathrm{D} 2 \mathrm{R}$ reduces dopamine concentration in the striatum and in turn exerts overall dopamine receptor hypo-stimulation coupled to motor depression in mice $^{31,32,35}$. Interestingly, we found that regardless of genotype, the administration of quinpirole similarly inhibited motor exploration in a novel environment (Fig. 2a). These data indicate that a lack of mTOR does not affect normal presynaptic D2R receptor-dependent motor effects in animals.
Administration of typical antipsychotic drug (haloperidol $\left(0.5 \mathrm{mg} / \mathrm{kg}\right.$, i.p.), which inhibits post-synaptic D2R ${ }^{35-37}$, robustly induced catalepsy in the control groups (Fig. 2b, c). Conversely, haloperidol administration completely failed to induce any cataleptic effect in $m T O R$ mutant mice (Fig. 2b, c). Thus, a striking and complete loss of haloperidol-induced extrapyramidal symptoms was observed in the mTOR mutant mice (Fig. 2b, c). These results indicate that $m T O R$ depletion interrupts the haloperidol-induced cataleptic effect, suggesting that mTOR signaling selectively controls post-synaptic D2R signaling in the striatal MSNs.

\section{mTOR mediates haloperidol-induced pS6 phosphorylation in the striatum}

Because haloperidol-induced catalepsy is completely abolished in the mTOR mutant mice, we hypothesized that haloperidol might promote mTOR signaling in the striatum. We administered haloperidol to mTOR mutant mice and the control group and isolated striatal tissue after $20 \mathrm{~min}$. We found a clear upregulation of pS6 (S235/ 236) by haloperidol only in the AAV-GFP-control group but not in the mTOR mutant mice (Fig. 2d, e). Surprisingly, haloperidol did not induce the phosphorylation of S6K or the 4EBP1, which are the direct mTORC1 targets. These data are consistent with a previous report ${ }^{38}$. Although the reasons for this are unclear, it was proposed that the basal S6K activity may be sufficient to induce pS6 because deletion of S6K abolishes haloperidol-induced pS6 in the striatum ${ }^{39}$. Phosphoinositide-3 kinase target pAkt (T308) signaling or mTORC2 target pAkt (S473) 


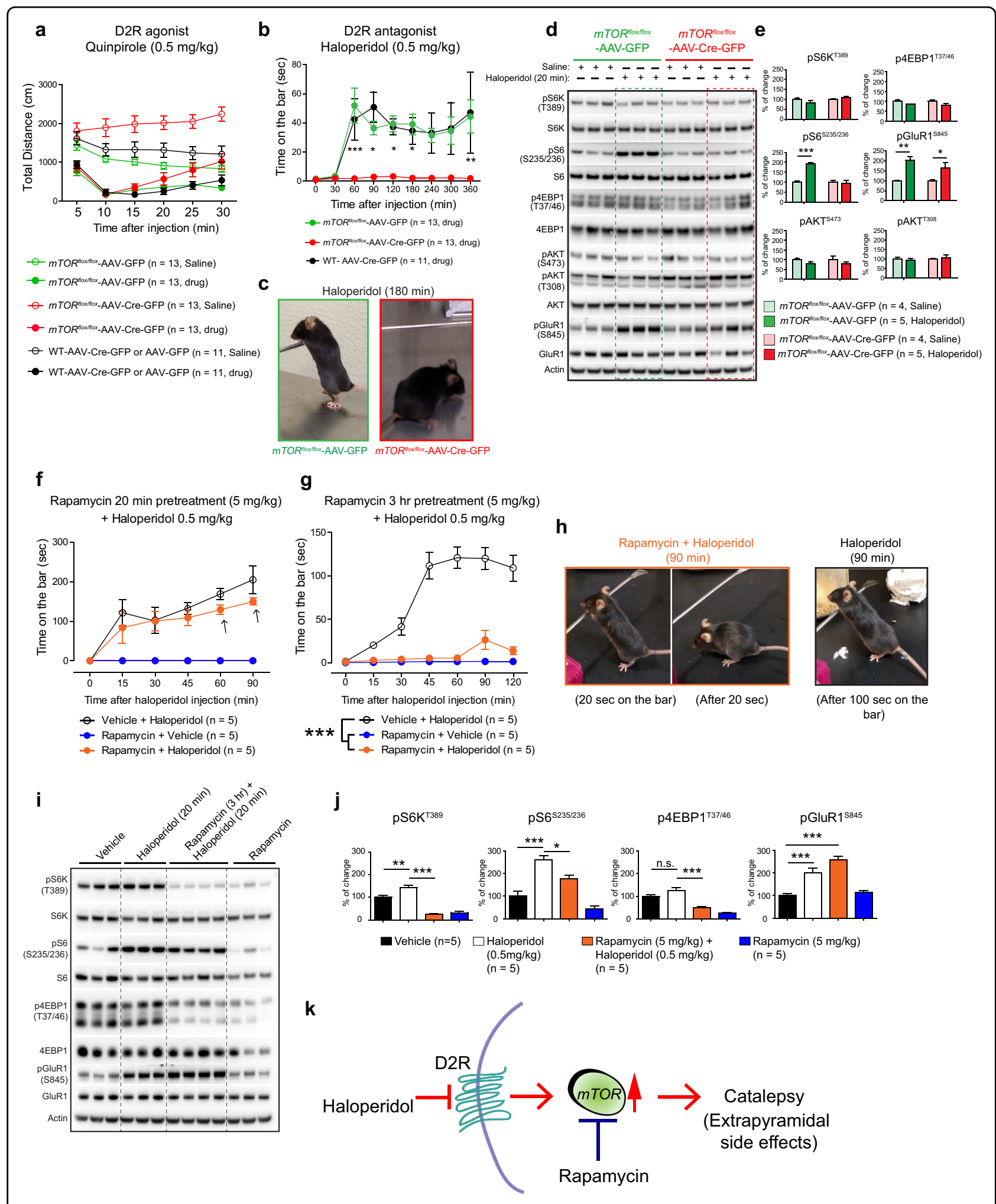

Fig. 2 (See legend on next page.) 
(see figure on previous page)

Fig. 2 mTOR depletion abolishes D2R antagonist haloperidol-induced catalepsy. a D2R agonist quinpirole (0.5 mg/kg, i.p.)-induced open-field activity, in AAV-Cre-GFP or AAV-GFP-injected $m T O R^{\text {floxflox }}$ mice and WT control mice. Data are mean \pm SEM, repeated measures two-way ANOVA followed by Bonferroni post-hoc test. b Catalepsy (as measured by time on the bar)-induced by D2R antagonist haloperidol (0.5 mg/kg, i.p.) in AAVCre-GFP or AAV-GFP-injected $m T O R^{\text {floxflox }}$ and WT control mice. Data are mean $\pm \mathrm{SEM},{ }^{*} P<0.05,{ }^{* *} P<0.01,{ }^{* * *} P<0.001$, repeated measures two-way ANOVA followed by Bonferroni post-hoc test. c Representative image of AAV-Cre-GFP or AAV-GFP-injected $m T O R^{\text {floxflox }}$ mice treated with haloperidol $(180 \mathrm{~min}) \cdot \mathrm{mTOR}^{\text {flox }}$ flox mice injected with AAV-GFP $(n=13$, female $=10$, male $=3)$, AAV-Cre-GFP $(n=13$, female $=6$, male $=7)$ or WT mice injected with AAV-GFP or AAV-Cre-GFP $(n=11$, female $=5$, male $=6)$ were treated with vehicle or drug $(\mathbf{a}-\mathbf{c})$. $\mathbf{d}$ Western blot analysis of indicated proteins from striatum of indicated mice after $20 \mathrm{~min}$ of haloperidol $(0.5 \mathrm{mg} / \mathrm{kg}$, i.p.) or saline injection. e Bar graph indicates quantification of the indicated proteins from (d). Data are mean $\pm \mathrm{SEM}, n=4$ for saline injected group and $n=5$ for haloperidol treated group, ${ }^{*} P<0.05,{ }^{* *} P<0.01$, ${ }^{* * *} P<0.001$, two-way ANOVA, Bonferroni post-hoc test. f, $\mathbf{g}$ Quantification of catalepsy induced by D2R antagonist haloperidol $(0.5 \mathrm{mg} / \mathrm{kg}$, i.p.) in vehicle or pretreated with rapamycin (5 mg/kg, i.p.) for $20 \mathrm{~min}(\mathbf{f})$ or $3 \mathrm{hr}(\mathbf{g})$ in C57BL/6 WT mice. Data are mean $\pm \mathrm{SEM}, n=5$ per group, ${ }^{* * *} P<0.001$, repeated measures two-way ANOVA, Bonferroni post-hoc test. $\mathbf{h}$ Representative image of haloperidol-induced catalepsy in WT mice pretreated with rapamycin or vehicle. $\mathbf{i}, \mathbf{j}$ Western blot analysis (i) and quantification (j) of indicated targets from the striatal tissue after 20 min of haloperidol and rapamycin pretreatment ( 3 hr). Data are mean $\pm \mathrm{SEM}, n=5$ per group, ${ }^{*} P<0.05,{ }^{* *} P<0.01,{ }^{* * *} P<0.001$, two-way ANOVA, Bonferroni post-hoc test. $\mathbf{k}$ Model shows mTOR mediates D2R inhibitory signals to induce catalepsy linked to extrapyramidal side effects in humans.

was also unaltered in the striatum of the treatment and control groups (Fig. 2d, e).

It is well known that by blocking D2R, haloperidol unmasks the ability of adenosine A2AR to enhance striatal cAMP/PKA signaling and ultimately increases the phosphorylation levels of the glutamate receptor subunit GluR1 [pGluR1 (S845) $]^{30,38}$. Interestingly, we found that haloperidol robustly induced pGluR1 (S845) in all genotypes and that the extent of activation was similar between mTOR mutant and control animals (Fig. 2d, e). Thus, mTOR does not mediate haloperidol-induced pGluR1 signaling in the striatum.

As A2AR and D2R knockout mice showed diminished haloperidol-induced catalepsy ${ }^{40,41}$ and because haloperidol acts by blocking of D2R, we wanted to confirm that striatal expression of these receptors was comparable in the mTOR mutant mice and control mice. We found similar A2AR levels (but significantly enhanced D2R levels) in the striatum of the mTOR mutant mice compared to the control (Supplementary Fig. 1). Thus, diminished haloperidol-induced catalepsy is not due to diminished A2AR or D2R levels in the mTOR mutant mice.

\section{Pharmacological inhibition of mTOR abolishes haloperidol- induced catalepsy}

As striatal genetic depletion of mTOR completely abolished the haloperidol-induced catalepsy, we next asked whether pharmacological inhibition of mTOR would produce a similar phenotype. To investigate this, we treated 4-month-old C57BL/6 WT mice with mTORC1 inhibitor rapamycin $(5 \mathrm{mg} / \mathrm{kg}$., i.p.) for $20 \mathrm{~min}$, followed by injection with haloperidol $(0.5 \mathrm{mg} / \mathrm{kg}$, i.p.). Haloperidol promoted a time-dependent cataleptic behavioral response in the vehicle-injected C57BL/6 mice, as well as in the rapamycin pretreated C57BL/6 WT mice (Fig. 2f). As expected, rapamycin treatment alone did not elicit a catalepsy response (Fig. 2f). This result indicates that 20 min of pretreatment with rapamycin does not affect the haloperidol-induced cataleptic response.

Interestingly, although the onset of the cataleptic behavioral response was similar between the groups, there was a trend towards decreased cataleptic behavior in the rapamycin pretreated animals after $60-$ and $90-\mathrm{min}$ post haloperidol administration (Fig. 2f, arrow). This observation prompted us to hypothesize that rapamycin may interfere with a cataleptic response after $60 \mathrm{~min}$ or longer duration following administration. To investigate this hypothesis, we pretreated C57BL/6 WT mice with rapamycin $(5.0 \mathrm{mg} / \mathrm{kg}$., i.p.) for $3 \mathrm{~h}$ before administering haloperidol $(0.5 \mathrm{mg} / \mathrm{kg}$, i.p.). Strikingly, we found a dramatic attenuation of haloperidol-induced catalepsy in animals that were pretreated with rapamycin for $3 \mathrm{~h}$ (as compared to vehicle-treated groups) (Fig. 2g, h). This result indicates that a more prolonged exposure to rapamycin [which may be necessary for target (mTOR) engagement] is a prerequisite to block the haloperidolinduced cataleptic response in mice.

\section{Pharmacological inhibition of mTOR diminishes haloperidol-induced pS6K, pS6 but not pGluR1}

Next, we investigated how rapamycin pretreatment impacted on haloperidol-induced striatal signaling in C57BL/6 WT mice. Compared to the vehicle, we found that haloperidol (for $20 \mathrm{~min}$ ) robustly induced striatal pS6 (S235/236) and pGluR1 (S845) signaling in C57BL/6 WT mice (Fig. 2i, j). Haloperidol did not induce p4EBP1 (T37/ 46) in C57BL/6 WT mice, consistent with genetic model (Fig. 2d, e). However, a slight but significant increase of pS6K (T389) (Fig. 2i, j) was observed in haloperidol injected C57BL/6 WT mice. Rapamycin pretreatment suppressed the haloperidol-induced pS6K and pS6 as well as diminished the basal pS6K, pS6, and p4EBP1. Rapamycin did not interfere with pGluR1 signaling, in the striatum (Fig. 2i, j), consistent with the observation in genetic model (Fig. 2d, e). These results indicate that 
pharmacological blocking of mTORC1 by rapamycin prevents the haloperidol-mediated mTORC1 signaling and associated catalepsy in the striatum.

\section{Discussion}

The data presented here indicate that mTOR signaling in the striatum mediates post-synaptic D2R-mediated functions, as both genetic depletion of mTOR or pharmacological inhibition of mTORC1 signaling by rapamycin prevented a haloperidol-induced catalepsy response (Fig. 2k). Importantly, mTOR regulates specific signaling and behavioral functions in the striatum. The D1R-mediated motor behaviors and the presynaptic D2R signaling are unaffected by the loss of striatal mTOR. Our data represent, to the best of our knowledge, the first report to use rapamycin to assess the role of mTOR signaling in haloperidol-induced catalepsy.

Previous studies showed that haloperidol induces pS6 signaling by enhancement of adenosine A2A/Golf signaling; however, the functional relevance of this pathway and its role in cataleptic behaviors were unknown $^{38,42}$. PKA signaling that induces pGluR1 is particularly linked to the generation of haloperidolinduced catalepsy ${ }^{43,44}$. Studies in non-neuronal cells showed that PKA acts upstream of mTOR and can activate or inhibit it ${ }^{45-47}$. PKA can directly phosphorylate mTOR and promote the phosphorylation of S6K in adipose tissue ${ }^{48}$. Indeed, it has been demonstrated that PKA activation induces pS6 in cultured striatal neurons ${ }^{38}$. With rapamycin or mTOR depletion, we found that mTOR signaling in the striatum did not interfere with haloperidol-induced pGluR1 signaling; however, it altogether abolished haloperidol-induced catalepsy. Thus, our data indicate that mTOR signaling in the striatum promotes haloperidol-induced catalepsy by acting downstream or independently of PKA-pGluR1 signaling.

The results presented here clearly suggest that an acute pretreatment of rapamycin completely reverses haloperidol-induced catalepsy, further emphasizing the critical role of mTORC1 in altering D2R signaling to promote extrapyramidal symptoms. Note that short-term (20 min) pretreatment with rapamycin had a negligible effect. However, long-term rapamycin pretreatment (3 hrs) abolished haloperidol-induced catalepsy. One possibility for such delayed action is due to the relatively poor brain penetrability of rapamycin and thus a delayed target engagement ${ }^{49}$. Interestingly, a previous study indicated Fyn kinase had a role in the regulation of haloperidol-induced catalepsy ${ }^{50}$. Fyn kinase also promotes mTORC1 signaling and it is therefore tempting to speculate that Fyn-mTORC1 signaling may have a role in haloperidol-mediated catalepsy ${ }^{50,51}$.

What are the molecular mechanisms underlying haloperidol-mTORC1-cataleptic behavior? A previous study indicated that haloperidol induced the mTORdependent translation and neuronal morphology in cultured $\mathrm{MSNs}^{52}$. In vivo, haloperidol can increase or decrease MSN morphology (spine density); in particular, it can decrease the spine density in D2 $\mathrm{MSN}^{37}$. Therefore, it is conceivable that mTOR is a critical regulator of haloperidol-induced molecular changes in the striatum. In addition to protein synthesis, mTOR signaling also regulates autophagy, purine, and lipid biosynthesis ${ }^{53}$. Based on our study, it is possible that mTOR signaling may translate the haloperidol-induced signaling into catalepsy by more than one pathway. Further research on the importance of these mechanistic insights, by dissecting the cell-type-specific role of mTOR, identification of haloperidol-induced mTOR interactors, and highthroughput comparative proteomic analysis in mTOR mutant and WT mice, could help unravel D2R-specific mechanisms of mTOR signaling in extrapyramidal symptoms.

Haloperidol is a major antipsychotic medication prescribed to diminish psychosis in schizophrenia patients ${ }^{54}$. However, its action is limited due to its elicitation of Parkinsonian-like bradykinesia, which affects a majority of patients and is commonly called haloperidol-induced $\mathrm{EPS}^{55,56}$. To date, there are no effective treatments available for haloperidol-induced EPS. By combining genetic and pharmacological approaches, our mechanistic models provide a clear insight into the causal role of mTOR signaling in promoting haloperidol-induced catalepsy in preclinical murine systems (Fig. 2k). Psychomotor abnormalities have long been observed in patients with mental disorders, such as ASD, SCZ, anxiety bioplar and depression, but the neurobiological mechanisms remain unclear. Our study therefore illustrates that mTOR is a critical regulator of motor abnormalities in neurological disorders and the translational potential of rapamycin in alleviating striatal D2R-mediated EPS in humans.

\section{Materials and methods \\ Chemicals and Antibodies}

The majority of the chemicals used were purchased from Sigma, unless mentioned otherwise. Antibodies against-mTOR (\#2983) pS6K T389 (\#9234), S6K (\#9202), pS6 S235/236 (\#4858), S6 (\#2217), p4EBP1 T37/46 (\#2855), 4EBP1 (\#9644), pAkt S473 (\#4060), pAkt T308 (\#13038), and Akt (\#4691) were from Cell Signaling Technology. Antibodies for actin (sc-47778), A2AR (sc32261), and D2R (sc-5303) were from Santa-Cruz Biotechnology. Ctip2 (ab18465) antibody was from Abcam. GFP antibody (AB3080), (+/-)-Quinpirole dihydrochloride (Q111), Haloperidol (H1512), and R(+)-SCH23390 hydrochloride (D054) were purchased from MilliporeSigma. SKF 81297 hydrobromide (1447) was from R\&D systems. Rapamycin (R-5000) was purchased from 
LC laboratories . Haloperidol was initially dissolved in glacial acetic acid, then its $\mathrm{pH}$ was adjusted close to 7 with $\mathrm{NaOH}$, and final dilution was made in saline solution $(0.9 \%)$. Rapamycin was dissolved in 5\% dimethyl sulfoxide (DMSO), 15\% PEG-400 (polyethylene glycol, molecular weight 400), and 5\% Tween-20, and finally dissolved in saline solution for injection. SCH23390, SKF81297, and Quinpirole were dissolved in saline solution. All the drugs were administrated by intraperitoneal (i.p.) injection.

\section{Animals}

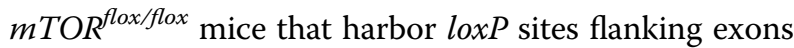
1-5 of the mTOR locus (The Jackson Laboratory, strain B6.129S4-mTOR ${ }^{t m 1.2 K o z} / J$, Stock No: 011009) and C57BL/ 6J (wild type, WT) were used for adeno-associated virus micro-injections. Mice were housed in groups of two or three on a 12:12 h light-dark cycle and were provided food and water ad libitum. All protocols were approved by Institutional Animal Care and Use Committee at The Scripps Research Institute, Florida,

\section{Stereotaxic surgeries}

For all surgical procedures, 8-week old mice were anesthetized through the constant delivery of isoflurane while mounted in a stereotaxic frame (David Kopf Instruments). AAV-Cre-GFP (AAV1.hSyn.HI.eGFP-Cre. WPRE.SV40) or AAV-GFP (green fluorescent protein, AAV1.hSyn.eGFP.WPRE.bGH) (Vector Core, University of Pennsylvania) were injected bilaterally into the striatum at the following coordinates: $\mathrm{ML}= \pm 1.6, \mathrm{AP}=+1.1$; $\mathrm{DV}=-3.9 /-3.5$ and $\mathrm{ML}= \pm 2.5 ; \mathrm{AP}=+0.5$; and $\mathrm{DV}=$ $-4.2 /-3.6$ from bregma. Virus was injected in $0.5 \mu \mathrm{l}$ volumes $\left(5.9 \times 10^{12} \mathrm{gc} / \mathrm{mL}\right)$ per injection site in each animal $(4 \mu \mathrm{l}$ total). Animals recovered for 2 weeks before behavioral testing. The efficacy of the viral injections was determined by GFP expression in the striatum.

\section{Behavioral analysis}

Longitudinal behavioral testing was performed for AAV-Cre-GFP or AAV-GFP-injected $m T O R^{\text {flox/flox }}$ mice, and wild type (WT) mice. All behavioral testing was performed as described in our previous work ${ }^{57,58}$ during the light phase of the light-dark cycle between 8:00 am and 12:00 pm. For each week/month of behavioral testing, the following measures were assessed with the rotarod test on the first four days and an open-field test on the fifth. Rotarod testing was performed using a linear accelerating rotation paradigm (Med Associates Inc.) for three trials separated by $20 \mathrm{~min}$ for four consecutive days each month. The mice were placed on the apparatus at $4 \mathrm{rpm}$ and were subjected to increasing rpm, accelerating to $40 \mathrm{rpm}$ over the course of a maximum of $5 \mathrm{~min}$. The overall latency to fall for each mouse was calculated as the average of the three trials across four days for each month.
The latency of falling from the rod was scored as an index of motor coordination, while improvement in performance across training days, as measured by increasing latency to fall from the rotarod, indicates motor learning. Open-field activity was assessed in a single 30-min session using EthoVision XT software (Noldus Information Technology). Each mouse was placed individually in the center of each square enclosure, and movement was quantified automatically. Single cohort of mice with mixed sex ratio were used for the behavior testing of AAV injected $m T O R^{f l o x / f l o x}$ mice: $m T O R^{f l o x / f l o x}$-AAV-GFP $(n=$ 13 , male 3 and female 10) and $m T O R^{\text {flox/flox }}$-AAV-CreGFP ( $n=13$, male 7 and female 6$)$. Single cohort of mice were used for behavior testing of AAV injected WT mice: WT-AAV-GFP $(n=5$, female 2 , male 3$)$ and WT- AAVCre-GFP $(n=6$, female 3 , male 3$)$. As there were no differences in the behaviors of WT-GFP and WT-CreGFP-injected mice, they were combined for the group analysis. The sample size was determined based on our previous studies ${ }^{31,32}$. For the pharmacological studies, animals were randomly assigned to vehicle or drug treatment before the experiment. All the behavioral tests were carried out in a blinded manner.

\section{Quinpirole and SKF81297 evaluation}

Pharmacological effect of D2R agonist Quinpirole and D1R agonist SKF81297 was made using the same Openfield system and the EthoVision XT software (Noldus Information Technology). Each mouse was placed individually in the center of a plastic box $(11 \times 14$ inches $)$ with fresh bedding. For SKF81297 evaluation, mice were placed in the boxes for $30 \mathrm{~min}$, as basal activity and habituation, then the drug was injected $(2.5 \mathrm{mg} / \mathrm{kg}$, i.p. $)$ and the total activity was recorded for $90 \mathrm{~min}$. Results were plotted in a bar-graph showing the \% change in the total activity after habituation at 0,30 , and $60 \mathrm{~min}$. For Quinpirole experiment, mice were placed in the center of the plastic box with fresh bedding after the drug injection $(0.5 \mathrm{mg} / \mathrm{kg}$, i.p.), then the total distance traveled $(\mathrm{cm})$ was measured each $5 \mathrm{~min}$ during the next $30 \mathrm{~min}$. Before each protocol, animals were kept in a waiting room for at least $30 \mathrm{~min}$. Each control group was treated with the vehicle according to the drug.

\section{Haloperidol and SCH23390 evaluation}

Behavioral evaluation for D2R antagonist Haloperidol $(0.5 \mathrm{mg} / \mathrm{kg}$, i.p. $)$ and D1R antagonist SCH23390 $(0.1 \mathrm{mg} /$ $\mathrm{kg}$, i.p.) was made by measuring the catalepsy-induced effect using the bar test. Catalepsy was measured by placing each mouse with its forelegs on the bar in a kangaroo posture (Fig. $1 \mathrm{l}$ and 2c, h), latency to change the corporal posture was recorded for three trials, and average of them was used for group analysis. After drug injection mice were evaluated on the bar at 15, 30, 60, $90 \mathrm{~min}$ for 
SCH23390, and at 0, 30, 60, 90, 120, 180, 240, 300, and $360 \mathrm{~min}$ for Haloperidol in the $m T \mathrm{TOR}^{\text {flox/flox }}$ and WT mice injected with AAV-GFP or AAV-Cre-GFP viruses. For Rapamycin and Haloperidol experiments in C57BL/6 WT mice, animals were injected with Rapamycin $(5 \mathrm{mg} / \mathrm{kg}$, i.p.) as pretreatment at $20 \mathrm{~min}$ or $3 \mathrm{~h}$ before Haloperidol $(0.5 \mathrm{mg} / \mathrm{kg}$, i.p.). After Haloperidol injection mice were tested on the bar at 15, 30, 45, 60, 90, and $120 \mathrm{~min}$. Before each protocol, each mouse was kept in single cage in the procedure room for at least $30 \mathrm{~min}$. Each control group was treated with the vehicle according to the drug.

\section{Western blot analysis}

Twenty minutes after haloperidol injection, mice were euthanized by decapitation and brains were rapidly dissected and the striatum was quickly removed and snapfrozen in liquid nitrogen. Tissue was homogenized in RIPA buffer [50 mM Tris- $\mathrm{HCl}$ (pH 7.4), $150 \mathrm{mM} \mathrm{NaCl}$, $1.0 \%$ Triton X-100, $0.5 \%$ sodium deoxycholate, $0.1 \%$ SDS,) with a protease inhibitor cocktail (Roche, Sigma) and phosphatase inhibitors (PhosSTOP, Roche, Sigma). Protein concentration was measured using BCA protein assay reagent (Pierce). Protein lysates were loaded and separated by $4-12 \%$ Bis-Tris Gel (ThermoFisher Scientific), transferred to PVDF membranes, and probed with the indicated antibodies. Secondary antibodies were HRP-conjugated (Jackson Immuno Research, Inc). Chemiluminescence was detected using WesternBright Quantum (Advansta) ECL reagent using a chemiluminescence imager (Alpha Innotech). Western blotting experiment was carried out as described previously ${ }^{57-61}$. Relative levels of all the proteins were normalized to actin and quantified using Image J. Relative levels of phosphorylated proteins were normalized to respective normalized total proteins and quantified.

\section{Immunohistochemistry and analysis}

Immunostaining was performed as previously described $^{58,61,62}$. Briefly, mouse brains were fixed in $4 \%$ paraformaldehyde for overnight, cryoprotected in a sucrose/ PBS gradient at $4{ }^{\circ} \mathrm{C}(10,20$, and $30 \%)$, and embedded in Tissue-Tek OCT compound (Sakura). Coronal sections $(20 \mu \mathrm{m})$ were collected on Superfrost/Plus slides and immunostained after heat-induced antigen retrieval [10 min in boiling citrate buffer (pH 6.0), MilliporeSigma, C9999]. Primary antibodies used in this study were antiCtip2 (1:500, Abcam, ab18465), anti-mTOR (1:250, Cell Signaling, \#2983), and anti-GFP (Santa Cruz, sc-9996). Alexa Fluor 488, 594, and 647 conjugated secondary antibodies (ThermoFisher Scientific) were used in this study. Immunofluorescent brain sections were counterstained with DAPI and mounted using Fluoromount-G mounting medium (ThermoFisher Scientific). Images were obtained with the Zeiss LSM 880 microscope and processed using the ZEN software (Zeiss).
For cell quantification, five regions of interest (ROIs) of $100 \mu \mathrm{m}^{2}$ were defined in immunostained sections (four to five sections for each mouse, $n=4$ mice per group) of the medial striatum from $m T O R^{\text {flox/flox }}$ injected with AAVGFP or AAV-Cre viruses. Total number of cells were calculated by counting the DAPI stained nuclei. AAVInfected neurons were identifying by expression of the GFP. GFP expression was observed in the soma of the AAV-GFP infected neurons while AAV-Cre infected neurons expressed GFP in the nucleus. Percentage of the Ctip2, mTOR and GFP triple-positive neurons were determined considering DAPI stained cells as $100 \%$. The lateral ventricular area was determined in hematoxylin/ eosin-stained sections, from the same animals Four rostral $(+1.1$ from Bregma) and caudal $(+0.5$ from Bregma) sections from each animal $(n=4$ mice per group) were taken using the Leica DM5500B microscope. The lateral ventricular area was calculated by analyzing the images using the ImageJ software.

\section{Statistical analysis}

Data are presented as mean \pm SEM as indicated. All data sets were first checked for normality using the Kolmogorov-Smirnov normality test. Variance was found to be similar between the groups tested. Statistical analysis was performed with a Student's $t$ test or two-way analysis of variance (ANOVA) followed by Bonferroni post-hoc test or repeated measure two-way ANOVA followed by Bonferroni post-hoc test as indicated in the figure legends. Repeated measures two-way ANOVA where time was the repeated measure and treatment/genotype group was the fixed effect. Post-hoc Bonferroni multiple comparison tests were used to identify statistically significant differences between treatment/genotype groups at each time point. Significance was set at $P<0.05$. All statistical tests were performed using Prism 7.0 (GraphPad software).

\begin{abstract}
Acknowledgements
The authors would like to thank Melissa Benilous for her administrative help and the members of the lab for their continuous support and collaborative atmosphere. This research was supported by funding from National Institutes of Health/National Institute of Neurological Disorders and Stroke grant R01NS087019-01A1, National Institutes of Health/National Institute of Neurological Disorders and Stroke grant R01-NS094577-01A1 and a grant from Cure Huntington Disease Initiative (CHDI) foundation.
\end{abstract}

\section{Author details}

'Department of Neuroscience, The Scripps Research Institute, Florida, Jupiter, Florida 33458, USA. ${ }^{2}$ Department of Environmental, Biological, and

Pharmaceutical Sciences and Technologies, University of Campania Luigi Vanvitelli, 81100 Caserta, Italy. ${ }^{3}$ Laboratory of Behavioral Neuroscience, CEINGE Biotecnologie Avanzate, 80145 Naples, Italy

\section{Author contributions}

S.S. conceptualized the project. U.N.R.J. designed and carried out all the work in TTOR $^{\text {flox/flox }}$ and related control mice. A.U. directed, and N.S. co-designed, pharmacological experiments with U.N.R.J. W.P. performed preliminary behavioral analysis. S.S. wrote the paper with input from the co-authors. 


\section{Conflict of interest}

The authors declare that they have no conflict of interest.

\section{Publisher's note}

Springer Nature remains neutral with regard to jurisdictional claims in published maps and institutional affiliations.

Supplementary Information accompanies this paper at (https://doi.org/ 10.1038/s41398-020-01014-x).

Received: 9 June 2020 Revised: 20 June 2020 Accepted: 3 July 2020 Published online: 02 October 2020

\section{References}

1. Laplante, M. \& Sabatini, D. M. mTOR signaling in growth control and disease. Cell 149, 274-293 (2012).

2. Bockaert, J. \& Marin, P. mTOR in brain physiology and pathologies. Physiol. Rev. 95, 1157-1187 (2015).

3. Stallone G., Infante B., Prisciandaro C. \& Grandaliano G. mTOR and aging: an old fashioned dress. Int. J. Mol. Sci. 20(11) 2774 (2019).

4. Hoeffer, C. A. \& Klann, E. mTOR signaling: at the crossroads of plasticity, memory and disease. Trends Neurosci. 33, 67-75 (2010).

5. Zeng, L. H., Rensing, N. R. \& Wong, M. The mammalian target of rapamycin signaling pathway mediates epileptogenesis in a model of temporal lobe epilepsy. J. Neurosci. 29, 6964-6972 (2009).

6. Troca-Marin, J. A., Alves-Sampaio, A. \& Montesinos, M. L. Deregulated mTORmediated translation in intellectual disability. Prog. Neurobiol. 96, 268-282 (2012).

7. Ravikumar, B. et al. Inhibition of mTOR induces autophagy and reduces toxicity of polyglutamine expansions in fly and mouse models of Huntington disease. Nat. Genet. 36, 585-595 (2004).

8. Malagelada, C., Jin, Z. H., Jackson-Lewis, V., Przedborski, S. \& Greene, L. A. Rapamycin protects against neuron death in in vitro and in vivo models of Parkinson's disease. J. Neurosci. 30, 1166-1175 (2010).

9. Caccamo, A., De Pinto, V., Messina, A., Branca, C. \& Oddo, S. Genetic reduction of mammalian target of rapamycin ameliorates Alzheimer's disease-like cognitive and pathological deficits by restoring hippocampal gene expression signature. J. Neurosci. 34, 7988-7998 (2014).

10. Grahn, J. A., Parkinson, J. A. \& Owen, A. M. The cognitive functions of the caudate nucleus. Prog. Neurobiol. 86, 141-155 (2008).

11. Sarkar, S. et al. A rational mechanism for combination treatment of Huntington's disease using lithium and rapamycin. Hum. Mol. Genet 17, 170-178 (2008).

12. Dehay, B. et al. Pathogenic lysosomal depletion in Parkinson's disease. J. Neurosci. 30, 12535-12544 (2010).

13. Crews, L. et al. Selective molecular alterations in the autophagy pathway in patients with Lewy body disease and in models of alpha-synucleinopathy. PLOS ONE 5, e9313 (2010).

14. Fox, J. H. et al. The mTOR kinase inhibitor Everolimus decreases $\mathbf{S 6}$ kinase phosphorylation but fails to reduce mutant huntingtin levels in brain and is not neuroprotective in the R6/2 mouse model of Huntington's disease. Mol. Neurodegener. 5, 26 (2010).

15. Rolls, E. T. Neurophysiology and cognitive functions of the striatum. Rev. Neurol. (Paris) 150, 648-660 (1994).

16. Graybiel, A. M. \& Grafton, S. T. The striatum: where skills and habits meet. Cold Spring Harb. Perspect. Biol. 7, a021691 (2015).

17. Nishi, A., Kuroiwa, M. \& Shuto, T. Mechanisms for the modulation of dopamine $d(1)$ receptor signaling in striatal neurons. Front Neuroanat. 5, 43 (2011).

18. Kuroiwa, M. et al. Phosphodiesterase 4 inhibition enhances the dopamine D1 receptor/PKAVDARPP-32 signaling cascade in frontal cortex. Psychopharmacol. (Berl.) 219, 1065-1079 (2012).

19. Herve, D. Identification of a specific assembly of the $\mathrm{g}$ protein golf as a critical and regulated module of dopamine and adenosine-activated cAMP pathways in the striatum. Front Neuroanat. 5, 48 (2011).

20. Fernandez-Duenas, V. et al. Revealing adenosine A2A-dopamine D2 receptor heteromers in Parkinson's disease post-mortem brain through a new alphascreen-based assay. Int J Mol Sci 2019; 20(14).
21. Klemm, W. R. Drug effects on active immobility responses: what they tell us about neurotransmitter systems and motor functions. Prog. Neurobiol. 32, 403-422 (1989).

22. Sheng, M. J., Lu, D., Shen, Z. M. \& Poo, M. M. Emergence of stable striatal D1R and D2R neuronal ensembles with distinct firing sequence during motor learning. Proc. Natl Acad. Sci. USA 116, 11038-11047 (2019).

23. Eshraghi, M. et al. RasGRP1 is a causal factor in the development of I-DOPAinduced dyskinesia in Parkinson's disease. Sci. Adv. 6, eaaz7001 (2020).

24. Bergeron, Y., Chagniel, L., Bureau, G., Massicotte, G. \& Cyr, M. mTOR signaling contributes to motor skill learning in mice. Front Mol. Neurosci. 7, 26 (2014).

25. Kugler, S., Kilic, E. \& Bahr, M. Human synapsin 1 gene promoter confers highly neuron-specific long-term transgene expression from an adenoviral vector in the adult rat brain depending on the transduced area. Gene Ther. 10, 337-347 (2003).

26. Rezai Amin, S. et al. Viral vector-mediated Cre recombinase expression in substantia nigra induces lesions of the nigrostriatal pathway associated with perturbations of dopamine-related behaviors and hallmarks of programmed cell death. J. Neurochem 150, 330-340 (2019).

27. Bostan, A. C. \& Strick, P. L. The basal ganglia and the cerebellum: nodes in an integrated network. Nat. Rev. Neurosci. 19, 338-350 (2018).

28. Durieux, P. F., Schiffmann, S. N. \& de Kerchove d'Exaerde, A. Differential regulation of motor control and response to dopaminergic drugs by D1R and D2R neurons in distinct dorsal striatum subregions. EMBO J. 31, 640-653 (2012).

29. Vitucci, D. et al. Rasd2 modulates prefronto-striatal phenotypes in humans and 'schizophrenia-like behaviors' in mice. Neuropsychopharmacology 41, 916-927 (2016).

30. Ghiglieri, V. et al. Rhes influences striatal CAMP/PKA-dependent signaling and synaptic plasticity in a gender-sensitive fashion. Sci. Rep. 5, 10933 (2015).

31. Usiello, A. et al. Distinct functions of the two isoforms of dopamine D2 receptors. Nature 408, 199-203 (2000).

32. Napolitano, F. et al. Role of aberrant striatal dopamine D1 receptor/CAMP/ protein kinase AVDARPP32 signaling in the paradoxical calming effect of amphetamine. J. Neurosci. 30, 11043-11056 (2010).

33. Morelli, M. \& Di Chiara, G. Catalepsy induced by SCH 23390 in rats. Eur. J. Pharm. 117, 179-185 (1985).

34. Napolitano, F. et al. The striatal-enriched protein Rhes is a critical modulator of cocaine-induced molecular and behavioral responses. Sci. Rep. 9, 15294 (2019).

35. Radl, D. et al. Differential regulation of striatal motor behavior and related cellular responses by dopamine D2L and D2S isoforms. Proc. Natl Acad. Sci. USA 115, 198-203 (2018).

36. Centonze, D. et al. Chronic haloperidol promotes corticostriatal long-term potentiation by targeting dopamine D2L receptors. J. Neurosci. 24, 8214-8222 (2004).

37. Sebel, L. E., Graves, S. M., Chan, C. S. \& Surmeier, D. J. Haloperidol Selectively Remodels Striatal Indirect Pathway Circuits. Neuropsychopharmacology 42, 963-973 (2017)

38. Valjent, E. et al. Haloperidol regulates the state of phosphorylation of ribosomal protein S6 via activation of PKA and phosphorylation of DARPP-32. Neuropsychopharmacology 36, 2561-2570 (2011).

39. Bonito-Oliva, A. et al. Haloperidol promotes mTORC1-dependent phosphorylation of ribosomal protein $\mathrm{S} 6$ via dopamine- and CAMP-regulated phosphoprotein of $32 \mathrm{kDa}$ and inhibition of protein phosphatase-1. Neuropharmacology 72, 197-203 (2013).

40. Boulay, D. et al. Haloperidol-induced catalepsy is absent in dopamine D(2), but maintained in dopamine $\mathrm{D}(3)$ receptor knock-out mice. Eur. J. Pharm. 391, 63-73 (2000)

41. El Yacoubi, M., Ledent, C., Parmentier, M., Costentin, J. \& Vaugeois, J. M. Adenosine A2A receptor knockout mice are partially protected against druginduced catalepsy. Neuroreport 12, 983-986 (2001).

42. Bowling, $\mathrm{H}$. et al. Antipsychotics activate mTORC1-dependent translation to enhance neuronal morphological complexity. Sci. Signal 7, ra4 (2014).

43. Adams, M. R. et al. Loss of haloperidol induced gene expression and catalepsy in protein kinase A-deficient mice. Proc. Natl Acad. Sci. USA 94, 12157-12161 (1997).

44. Roche, K. W., O'Brien, R. J., Mammen, A. L., Bernhardt, J. \& Huganir, R. L. Characterization of multiple phosphorylation sites on the AMPA receptor GluR1 subunit. Neuron 16, 1179-1188 (1996). 
45. Jewell J. L. et al. GPCR signaling inhibits mTORC1 via PKA phosphorylation of Raptor. Elife 8, e43038 (2019).

46. de Joussineau, C. et al. mTOR pathway is activated by PKA in adrenocortical cells and participates in vivo to apoptosis resistance in primary pigmented nodular adrenocortical disease (PPNAD). Hum. Mol. Genet. 23, 5418-5428 (2014).

47. Kim, H. W. et al. Cyclic AMP controls mTOR through regulation of the dynamic interaction between Rheb and phosphodiesterase 4D. Mol. Cell Biol. 30, 5406-5420 (2010).

48. Liu, D. et al. Activation of mTORC1 is essential for beta-adrenergic stimulation of adipose browning. J. Clin. Invest. 126, 1704-1716 (2016).

49. Brandt, C. et al. The novel, catalytic mTORC1/2 inhibitor PQR620 and the PI3K/ mTORC1/2 inhibitor PQR530 effectively cross the blood-brain barrier and increase seizure threshold in a mouse model of chronic epilepsy. Neuropharmacology 140, 107-120 (2018).

50. Hattori, K. et al. Fyn is required for haloperidol-induced catalepsy in mice. J. Biol. Chem. 281, 7129-7135 (2006).

51. Wang, Y., Yamada, E., Zong, H. \& Pessin, J. E. Fyn activation of mTORC1 stimulates the IRE1alpha-JNK pathway, leading to cell death. J. Biol. Chem. 290, 24772-24783 (2015).

52. Bowling, H. \& Santini, E. Unlocking the molecular mechanisms of antipsychotics - a new frontier for discovery. Swiss Med. Wkly 146, w14314 (2016).

53. Ben-Sahra, I. \& Manning, B. D. mTORC1 signaling and the metabolic control of cell growth. Curr. Opin. Cell Biol. 45, 72-82 (2017).
54. Ostinelli, E. G., Brooke-Powney, M. J., Li, X. \& Adams, C. E. Haloperidol for psychosis-induced aggression or agitation (rapid tranquillisation). Cochrane Database Syst. Rev. 7, CD009377 (2017).

55. Finucane, A. M. et al. Drug therapy for delirium in terminally ill adults. Cochrane Database Syst. Rev. 1, CD004770 (2020).

56. Kurz, M., Hummer, M., Oberbauer, H. \& Fleischhacker, W. W. Extrapyramidal side effects of clozapine and haloperidol. Psychopharmacology (Berl.) 118, 52-56 (1995).

57. Pryor, W. M. et al. Huntingtin promotes mTORC1 signaling in the pathogenesis of Huntington's disease. Sci. Signal 7, ra103 (2014).

58. Swarnkar, S. et al. Ectopic expression of the striatal-enriched GTPase Rhes elicits cerebellar degeneration and an ataxia phenotype in Huntington's disease. Neurobiol. Dis. 82, 66-77 (2015).

59. Shahani, N. et al. Rheb GTPase regulates beta-secretase levels and amyloid beta generation. J. Biol. Chem. 289, 5799-5808 (2014).

60. Shahani, N. et al. RasGRP1 promotes amphetamine-induced motor behavior through a Rhes interaction network ("Rhesactome") in the striatum. Sci. Signal 9, ra111 (2016)

61. Shahani, N., Huang, W. C., Varnum, M., Page, D. T. \& Subramaniam, S. Forebrain depletion of Rheb GTPase elicits spatial memory deficits in mice. Neurobiol. Aging 50, 134-143 (2017).

62. Chen, Y., Huang, W. C., Sejourne, J., Clipperton-Allen, A. E. \& Page, D. T. Pten mutations alter brain growth trajectory and allocation of cell types through elevated beta-catenin signaling. J. Neurosci. 35, 10252-10267 (2015). 